\title{
Actividad antimicrobiana del extracto hidroalcohólico de Calendula officinalis $\mathrm{L}$.
}

\author{
Rodrigo Rodríguez Cepeda ${ }^{1^{*}} ;$ Nelsy Yanet Alvarez Suarez ${ }^{2^{*}}$ \\ 1'Universidad Pedagógica Nacional, Cl. 72 \#11-86, Bogotá D.C. Colombia; ${ }^{2}$ Kolbe Internacional SAS, \\ Carrera 49B \#. 171A-84, Bogotá D.C. Colombia \\ *rrodriguez@pedagogica.edu.co
}

\begin{abstract}
Resumen
La búsqueda de productos naturales, con determinadas funciones como aditivos alimentarios, son de gran interés investigativo por su proyección industrial. Por esta razón, el presente documento muestra los resultados de un estudio exploratorio sobre la actividad antibacteriana de un extracto hidroalcohólico de Calendula officinalis $L$., el cual se caracterizó mediante análisis fitoquímico, espectroscopía InfrarrojaAttenuated Total Reflection (IR-ATR), y cromatografía de gases acoplada a masas (CG-MS). El poder antimicrobiano se determinó sobre Pseudomona aeruginosa, Staphyloccocus aureus, y Candida albicans. Finalmente, se realizó una aplicación sobre fresa (Fragaria chiloensis L.), con el fin de observar el efecto sobre la vida útil del fruto, esto en las condiciones ambientales de temperatura $20^{\circ} \mathrm{C}$ y humedad relativa $70 \%$. Los resultados muestran que el extracto hidroalcohólico es una mezcla de diversos compuestos químicos, con buenas propiedades como biocompuestos activos. Finalmente se evidencia la presencia de taninos, carotenoides y compuestos fenólicos, entre otros, los cuales, de acuerdo con el estudio de actividad antimicrobiana, mejoraron la vida útil de la F. chiloensis.
\end{abstract}

Palabras clave: Aditivos naturales; Alimentos; Antimicrobianos; Conservación. 


\title{
Antimicrobial activity of the hydroalcoholic extract of Calendula officinalis L.
}

\begin{abstract}
The current search for natural products, with some functions as food additives, are of great research interest due to their industrial projection. For this reason, this document shows the results of an exploratory study on the antibacterial activity of a hydroalcoholic extract of Calendula officinalis L., which was characterized by phytochemical analysis, Infrared Attenuated Total Reflection (IR-ATR) spectroscopy, and gas chromatography coupled to masses (CG-MS). The antimicrobial power was determined on Pseudomona aeruginosa, Staphyloccocus aureus, and Candida albicans. Finally, an application was made on strawberry (Fragaria chiloensis L.) to observe the effect on the useful life of the fruit, this in environmental conditions of temperature $20^{\circ} \mathrm{C}$ and relative humidity $70 \%$. The results show that the hydroalcoholic extract is a mixture of various chemical compounds, with properties as active biocomposites. Finally, the presence of tannins, carotenoids, and phenolic compounds, among others, which, according to the study of antimicrobial activity, improved the useful life of $F$. chiloensis $L$ is evidenced.
\end{abstract}

Keywords: Natural additives; Food; Antimicrobial; Conservation.

\section{Atividade antimicrobiano do extrato hidroalcoólico de Calendula Officinalis L.}

\begin{abstract}
Resumo
A busca por produtos naturais, com determinadas funções como aditivos alimentares, são de grande interesse de pesquisa devido ao seu projeto industrial. Por esse motivo, este documento mostra os resultados de um estudo exploratório sobre a atividade antibacteriana de um extrato hidroalcoólico de Calendula officinalis $L$., que foi caracterizado por análise fitoquímica, espectroscopia de infravermelhoreflexão total atenuada (IR-ATR) e cromatografia de gases acoplados a massas (CG-MS). O poder antimicrobiano foi determinado em Pseudomona aeruginosa, Staphyloccocus aureus e Candida albicans. Por fim, foi feita uma aplicação em morango (Fragaria chiloensis L.), a fim de observar o efeito na vida útil do fruto, esta em condições ambientais de temperatura de $20^{\circ} \mathrm{C}$ e umidade relativa de $70 \%$. Os resultados mostram que o extrato hidroalcoólico é uma mistura de diversos compostos químicos, com boas propriedades como biocompósitos ativos. Por fim, evidencia-se a presença de taninos, carotenóides e compostos fenólicos, entre outros, que, segundo o estudo da atividade antimicrobiana, melhoraram a vida útil de $F$. chiloensis.
\end{abstract}

Palavras-Chave: Aditivos naturais; Comida; Antimicrobiano; Conservação. 


\section{Introducción}

Los procesos de degradación de alimentos han estado relacionados con diversas alteraciones que conllevan a generar cambios en las características nutricionales, fisicoquímicas y organolépticas [1], alteraciones que pueden involucrar problemas de salud a quien consuma estos alimentos. Ante esta problemática, los procesos industriales han optado por utilizar algunos aditivos que permiten controlar las alteraciones químicas de los alimentos, como los antioxidantes, agentes bactericidas o bacteriostáticos que controlan la población de microorganismos; sin embargo, los aditivos utilizados tradicionalmente en la industria alimentaria, como el butil-hidroxitolueno (BHT), butil-hidroxianisol (BHA), terbutil-hidroquinona $(T B H Q)$, etoxiquina $(E Q)$ o el galato de propilo ( $E$ 310 ), han sido cuestionados debido a su toxicidad y posibles efectos carcinogénicos, razón por la cual, se limita su uso o simplemente son evitados por los consumidores [2,3].

En este contexto, la búsqueda de aditivos de origen natural, enfocados a sustituir los utilizados tradicionalmente, han despertado el interés de los investigadores, especialmente porque, en la mayoría de los casos, el consumidor los asumen como beneficiosos por sus bajos efectos en la salud, comparados con los productos sintéticos [4], de esta manera, es posible encontrar diversos estudios en los cuales se han probado extractos de plantas como antioxidantes y antimicrobianos, entre los que se encuentran: jojoba, gingseng o jengibre, algunos de ellos haciendo parte inclusive de los empaques, con resultados aceptables en función de prolongar la vida útil de los alimentos [5]. De acuerdo con la FAO, un aditivo alimentario se define como cualquier sustancia que no se consume normalmente como alimento, ni tampoco se usa como ingrediente básico en alimentos, y cuya adición intencionada al alimento con fines tecnológicos, en cualquiera de las fases de elaboración, resulte en un componente más del alimento que afecta sus características, en la misma definición se excluyen los contaminantes y las sustancias que mejoran las cualidades nutricionales [6].

En este contexto, algunos estudios han utilizado extractos hidroalcohólicos de plantas como los obtenidos de la Flor de Jamaica (Hibiscus sabdariffa L.), los cuales cuentan con algunas propiedades bioactivas como antioxidantes y bactericidas, estos extractos, al ser utilizados sobre carne molida, demostraron una amplia eficacia sobre microorganismos Escherichia Coli y bacterias aerobias indicadores de calidad en carne y derivados cárnicos [7,8, 9].

Por su parte, la Calendula officinalis L., es una planta de la familia de las Asteraceaes, la cual contiene flavonoides, fenoles y otros compuestos químicos, reconocidos por sus características bioactivas frente a ciertos microorganismos, identificados como clínicamente patógenos, tanto gram negativos como gram positivos. Algunas investigaciones han demostrado que los extractos de caléndula incorporados en tratamientos terapéuticos periodontales son una buena alternativa frente a la creciente resistencia de los patógenos periodontales [10].

Dentro de este marco ha de considerarse, algunos trabajos donde se recomienda identificar las moléculas responsables de la eficiencia antimicrobiana, así como, la determinación de la eficiencia de extractos obtenidos a partir de la Caléndula, frente a otros microorganismos indicadores de calidad alimentaria [10,11,12]. Por lo que se presume, podrían ser utilizados en alimentos altamente perecederos, como por ejemplo la fresa (Fragaria chiloensis L.) que en Colombia es un producto importante en la economía campesina, a tal punto que en 2013 se produjeron 42.453 toneladas, siendo Cundinamarca el departamento con mayor producción (22.562 toneladas), seguido por Antioquia (12.545 toneladas), Norte de Santander (3.360 toneladas) y Cauca con 2.808 toneladas [13], y que es un producto altamente perecedero debido a los problemas fitosanitarios, especialmente por la presencia de hongos, ocasionados, entre otros aspectos, por su alta actividad acuosa

Las anteriores consideraciones han permitido plantear el propósito del presente trabajo, el cual consistió en caracterizar químicamente en extracto hidroalcohólico de la Calendula officinalis L., y determinar su posible efecto antimicrobiano sobre Pseudomona aeruginosa, Staphyloccocus aureus y Candida albicans, microorganismos indicadores de calidad en alimentos [14, 15], así como el efecto sobre la formación de mohos en la fresa, Fragaria chiloensis L.

\section{Metodología}

\section{Preparación de la muestra}

Las flores de caléndula fueron recolectadas en diferentes puntos de comercialización en la ciudad 
de Bogotá, la muestra de análisis se obtuvo mediante un muestreo aleatorio hasta lograr $5 \mathrm{~kg}$ y se mezcló con el fin de homogenizarla. Posteriormente, las flores se lavaron con agua fresca y limpia, se secaron a $40{ }^{\circ} \mathrm{C}$ en estufa de aire recirculado [16], finalmente se realizó un cuarteo para reducir la muestra hasta obtener el peso suficiente para realizar los ensayos.

\section{Extracción}

Se prepararon cuatro extractos hidroalcohólicos de las flores de caléndula, obtenidos mediante percolación en etanol grado alimenticio a 30, 50, 70 y $90^{\circ} \mathrm{GL}$ [17]. Para ello se utilizaron $250 \mathrm{~g}$ de flores limpias, secas, cortadas en pequeños trozos y tamizadas en malla $\mathrm{N}^{\circ} 3$ US mesh $(6,73 \mathrm{~mm})$, las cuales se mantuvieron en contacto con las diferentes soluciones hidroalcohólicas, bajo agitación constante durante cinco días a temperatura ambiente $\left(20^{\circ} \mathrm{C}\right)$. La relación flores:solvente de extracción fue 1:10, de esta manera se asegura que el solvente cubre la totalidad de la muestra. Una vez finalizado el tiempo de contacto, se eliminaron los trozos de flor mediante filtrado, el líquido obtenido después de la extracción fue concentrado, mediante presión reducida en un rota-evaporador (Büchi modelo W240K). El etanol recuperado se descartó, y los extractos hidroetanólicos se almacenaron en la oscuridad, a condiciones de refrigeración $\left(4{ }^{\circ} \mathrm{C}\right)$, con el fin de evitar procesos de oxidación en los diferentes compuestos que conforman el extracto $[2,8]$. Como criterio de selección para realizar la caracterización fitoquímica, estructural, identificación de compuestos químicos y ensayo de bioactividad, se consideró el rendimiento del proceso, en términos del volúmen final de extracto obtenido después de la destilación.

\section{Caracterización química del extracto}

Una vez determinado el extracto a caracterizar, se realizaron pruebas fitoquímicas mediante la identificación cualitativa de los metabolitos secundarios presentes en el extracto hidroalcohólico. Las pruebas realizadas fueron: Shinoda para flavonoides, Rosenthaler para saponinas, Cloruro férrico y acetato de plomo para taninos, Fluorescencia U.V. para cumarinas, Liebermann - Buchard para triterpenos, Mayer y Dragendorff para alcaloides, ensayo con ácido clorhídrico e hidróxido de sodio para quinonas y prueba con cloroformo para carotenoides, según la metodología propuesta por $[2,18,19]$, todas las pruebas se realizaron por triplicado.

Con el fin de complementar el análisis fitoquímico, se realizó un análisis de caracterización estructural, mediante la técnica de espectroscopía infrarroja, teniendo en cuenta las siguientes condiciones: equipo I.R. - ATR marca Shimadzu (IRAffinity1S) MIRacle 10 (diamond/ZnSe), resolución $4 \mathrm{~cm}^{-1}$, detector DLATGS. Con el cual fue posible identificar bandas características de algunos grupos funcionales que corroboran los resultados obtenidos en los análisis fitoquímicos del extracto. Estos resultados, así como los reportes de otros investigadores, permiten suponer posibles características bioactivas de los componentes presentes en el extracto.

Posteriormente, se realizó una identificación de los compuestos químicos presentes en el extracto hidroalcohólico, para lo cual se recurrió al método analítico de cromatografía de gases acoplada a masas, en un cromatógrafo de gases Agilent 6890 acoplado a un espectrómetro de masas Agilent 5973, las condiciones cromatográficas utilizadas fueron: Hélio como gas de arrastre; columna de sílica $0,53 \mathrm{~mm}^{*} 30 \mathrm{~m}^{*} 0,30 \mu \mathrm{m}$; temperatura del inyector $220^{\circ} \mathrm{C}$; y gradiente de temperatura en la columna $100^{\circ} \mathrm{C}$ durante $4 \mathrm{~min}$.; velocidad de calentamiento $50{ }^{\circ} \mathrm{C} / \mathrm{min}$. a $120^{\circ} \mathrm{C}$, después de 10 $\min .50^{\circ} \mathrm{C} / \mathrm{min}$. hasta $220^{\circ} \mathrm{C}$ durante $6 \mathrm{~min}$.

\section{Ensayos microbiológicos}

Una vez identificados los compuestos potencialmente bioactivos, se realizó el ensayo de efectividad antibacteriana del extracto frente a microorganismos indicadores, para esta investigación se utilizaron: Candida albicans ATCC 14053, Pseudomonas aeruginosa ATCC 15442 y Staphylococcus aureus ATCC 6538, y se siguieron los pasos establecidos en la metodología establecida por la norma NTC 5150, para determinar la actividad bactericida de productos antisépticos y desinfectantes [20, 21].

El método consistió en medir con una pipeta 8,0 $\mathrm{mL}$ del extracto hidroalcohólico, se adicionó $1,0 \mathrm{~mL}$ de agua y $1,0 \mathrm{~mL}$ de suspensión bacteriana que contenía entre $1,5^{*} 10^{8}$ y $5^{*} 10^{8} \mathrm{ufc} / \mathrm{mL}$, se mezcló y se llevó a un baño termostatado a $20 \pm 1^{\circ} \mathrm{C}$, la actividad bactericida del producto se determinó para tiempos de contacto de 5, 10 y 15 minutos. Antes de finalizar los tiempos de contacto, se tomó una alícuota de $1,0 \mathrm{~mL}$ de la mezcla anterior y se llevó a un tubo de ensayo que contiene $8,0 \mathrm{~mL}$ de neutralizador (Polisorbato $80,30 \mathrm{~g} / \mathrm{l}$; lecitina $3 \mathrm{~g} / \mathrm{l}$ ) y $1,0 \mathrm{~mL}$ de agua, se mezcló e incubó a $20 \pm 1^{\circ} \mathrm{C}$ por 
5 minutos, se incluyeron los controles positivo y negativo siguiendo la recomendación de la norma NTC 5150 [18].

A finalizar el tiempo de neutralización, se tomó una alícuota de $1,0 \mathrm{~mL}$, por duplicado, y se llevó a una caja de Petri, se adicionó entre 12 y $15 \mathrm{~mL}$ de medio fundido Agar de Soja Triptona (TSA). Posteriormente, se incubaron las placas a 36 o 37 $\pm 1{ }^{\circ} \mathrm{C}$ durante 24 horas, después de las cuales se realizó el conteo de unidades formadoras de colonia, nuevamente se incubó por 24 horas y se realizó el conteo de la placa solamente en aquellas donde las colonias estén bien separadas. Se determinó el número más alto de colonias por cada placa y se calculó el número de ufc/mL [18]. Finalmente, se realizó un ensayo sensorial exploratorio de efectividad, mediante la aplicación directa del extracto hidroalcohólico de la caléndula sobre fresas frescas y se evaluó visualmente el crecimiento de hongos. La aplicación del extracto se desarrolló mediante aspersión, con el fin de observar el efecto sobre la infestación por hongos, los cuales causan la llamada pudrición gris a un amplio rango de plantas y frutos. El ensayo por aspersión se efectuó siguiendo un diseño experimental completamente al azar, con cinco repeticiones y un testigo, al cual no se le adicionó el extracto, las condiciones ambientales del experimento fueron controladas en una temperatura de $20{ }^{\circ} \mathrm{C}$ y $70 \%$ de humedad relativa, las cuales favorecen el crecimiento de hongos, algunos experimentos reportan que la temperatura óptima de crecimiento es $24^{\circ} \mathrm{C}$, aun cuando puede soportar temperaturas cercanas a $0{ }^{\circ} \mathrm{C}$.

El efecto del extracto sobre el fruto se realizó mediante un seguimiento visual hasta observar el crecimiento de colonias en la superficie de la fresa, por lo que, el ensayo finalizó con la primera aparición clara de las colonias de hongos.

Las fresas utilizadas en el ensayo se obtuvieron en la Vereda El Charquito, Corregimiento II del municipio de Soacha, Cundinamarca a $2.566 \mathrm{~m}$ sobre el nivel del mar, en $4^{\circ} 32^{\prime} 39^{\prime \prime} \mathrm{N}$; $74^{\circ} 16^{\prime} 36^{\prime \prime}$ $\mathrm{O}$; la madurez del fruto se estableció mediante el cociente entre los Grados Brix y la acidez titulable, expresada como porcentaje de ácido cítrico [22], en un rango entre 10 y 16 , lo cual permitió una relativa homogeneidad.

\section{Resultados y discusión}

\section{Obtención del extracto hidroalcohólico}

Los rendimientos obtenidos en el proceso de extracción hidroalcohólica de las flores de caléndula, mediante la metodología de percolación, en una relación flores:solución extractora de 1:10, después de cinco días de contacto y posterior concentración del extracto en un rotavapor, a los resultados obtenidos se les realizó un análisis estadístico de comparación de medias entre variables independientes, con el fin de establecer diferencias estadísticas entre ellas y seleccionar el extracto a caracterizar. En la Tabla 1 se presentan los resultados obtenidos.

Tabla 1. Rendimiento de extracción.

\begin{tabular}{cc}
\hline Solvente de Extracción & \% Promedio del Rendimiento \\
\hline Etanol $30^{\circ} \mathrm{GL}$ & $2,72 \pm 0,09$ \\
Etanol $50^{\circ} \mathrm{GL}$ & $3,53 \pm 0,14$ \\
Etanol $70^{\circ} \mathrm{GL}$ & $4,25 \pm 0,30$ \\
${\text { Etanol } 90^{\circ} \mathrm{GL}}$ & $4,34 \pm 0,83$ \\
\hline Extracto & Comparación de medias \\
\hline Etanol $30^{\circ} \mathrm{GL}$ & Estadístico t $_{\text {exp. }}$ \\
\hline Etanol $50^{\circ} \mathrm{GL}$ & $0,001^{*}$ \\
\hline Etanol $50^{\circ} \mathrm{GL}$ & $0,002^{*}$ \\
\hline Etanol $70^{\circ} \mathrm{GL}$ & 0,868 \\
\hline Etanol $70^{\circ} \mathrm{GL}$ & \\
\hline
\end{tabular}

* Existen diferencias significativas, ya que $t_{\text {exp. }} \leq t=0.05$ 
En la Figura 1 se muestra el extracto concentrado, el cual tiene como característica principal una coloración oscura y textura aceitosa.

Teniendo en cuenta el resultado estadístico de comparación de medias (Tabla 1), en el cual el rendimiento de extracción logrado con etanol a $70{ }^{\circ} \mathrm{GL}$ no tiene una diferencia estadística significativa con el de $90{ }^{\circ} \mathrm{GL}$, los ensayo de caracterización fitoquímica, el análisis estructural, identificación de compuestos, actividad antimicrobiana y la aplicación en fresa, se realizó únicamente con el extracto de etanol a $70^{\circ} \mathrm{GL}$, ya que el proceso se hace un poco menos costoso, si se proyecta un probable escalonamiento a producción planta piloto o industrial.
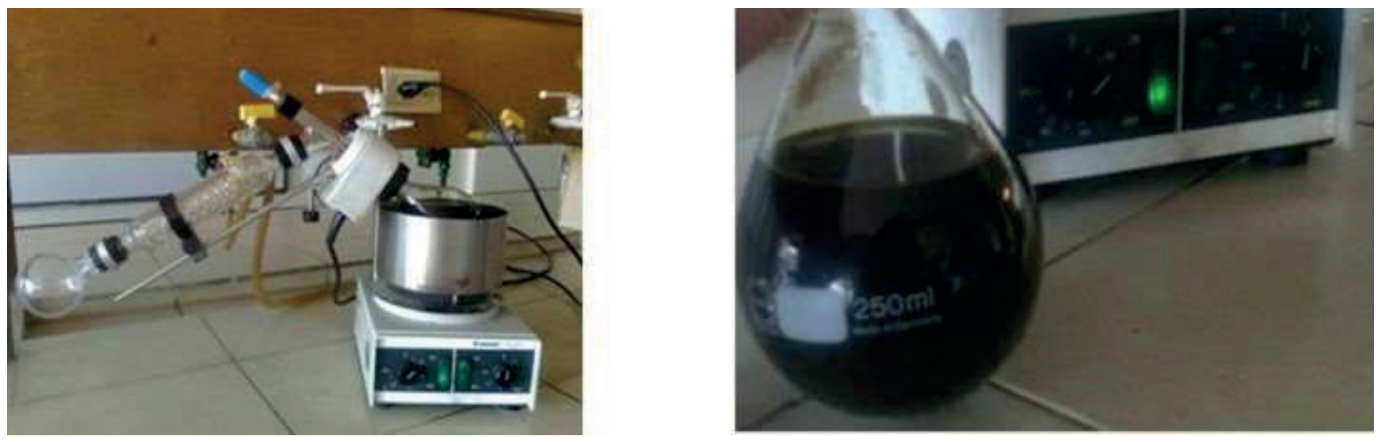

Figura 1. Extracto concentrado de caléndula.

Análisis de tamizaje fitoquímico

Los análisis fitoquímicos cualitativos realizados a los extractos concentrados de caléndula, obtenidos después de la percolación y eliminación del solvente, se resumen en la Tabla 2.

Tabla 2. Caracterización fitoquímica.

\begin{tabular}{|c|c|c|}
\hline Metabolito & Prueba & Resultado \\
\hline Flavonoides (Compuesto fenólico) & Shinoda & Positivo \\
\hline Saponinas & Rosenthaler & Positivo (coloración violeta) \\
\hline Taninos & Cloruro Férrico & Positivo \\
\hline Cumarinas & Fluorescencia U.V. & Positivo \\
\hline Triterpenos & Liebermann- Buchard & Positivo \\
\hline \multirow{2}{*}{ Alcaloides } & Mayer & Positivo \\
\hline & Dragendorff & Positivo \\
\hline Quinonas & Hidróxido de sodio & Positivo \\
\hline
\end{tabular}

Los resultados obtenidos evidencian el alto grado de complejidad del extracto hidroalcohólico, ya que es una mezcla de diversos compuestos químicos, todos ellos con buenas perspectivas como biocompuestos activos, así por ejemplo: a los flavonoides se les ha reconocido algunos efectos beneficiosos en la prevención de enfermedades cardiovasculares, neurodegenerativas o el cáncer [23]; los taninos han sido altamente estudiados y existe un amplio abanico de propiedades biológicas asociadas a estos compuestos, destacándose su capacidad antitumoral, fungicida, molusquicida, actividad hemolítica y antiinflamatoria, dependiendo de la diversidad estructural [24].
Por su parte, los taninos son compuestos polifenólicos a los que se les ha reconocido propiedades antitumorales, antimutagénicos, antidiabéticos, antioxidante y antibióticos, por lo que la presencia de estos compuestos puede favorecer la vida útil de alimentos, al ser utilizados como aditivos [25]. Las cumarinas son benzopironas que permiten modificar edemas, igualmente se le reconocen propiedades antiarrítmicas, antisépticas, analgésicas, antioxidantes e inclusive anticoagulantes, dependiendo en gran medida del tipo de cumarina presente $[2,26,27]$.

De otra parte, se ha relacionado la actividad anticancerígena, inmunomoduladora, 
antiinflamatoria, hipoglucemiante, hipolipemiante y hepatoprotectoras a la presencia de triterpenos, compuestos muy abundantes en el reino vegetal, estos son compuestos lipídicos no saponificables que se forman por la unión de isoprenos. Por su parte, los alcaloides son reconocidos por su actividad analgésica, así como compuestos con buenas propiedades para el tratamiento de enfermedades neurodegenerativas como el Alzheimer [28, 29].

Finalmente, el tamizaje fitoquímico evidencia la presencia de quinonas, las cuales, al igual que los anteriores compuestos, han sido materia de investigación por sus propiedades anticancerígenas, antiparasitarias, citotóxicos y por su capacidad de aceptar electrones, se les reconoce la propiedad de modular el estrés oxidativo, que es el mecanismo de inhibición en el crecimiento bacteriano, de virus y de células cancerígenas $[25,26]$.

La composición fitoquímica del extracto hidroalcohólico de la caléndula, evidencia su gran potencial como componente bioactivo a ser utilizado ya sea como antioxidante, bactericida, o como un aditivo alimentario con funciones preventivas de algunas enfermedades, lo cual justifica ampliar su estudio, mediante caracterización estructural y aplicación funcional en un alimento.

\section{Análisis infrarrojo}

Teniendo en cuenta que la caracterización fitoquímica realizada al extracto sugiere la presencia de compuestos con algunas propiedades importantes, se complementan los resultados obtenidos en el tamizaje fitoquímico, con un análisis infrarrojo, el cual permite identificar algunas señales propias de grupos funcionales representativos de las especies encontradas cualitativamente, como por ejemplo: la presencia de: alcanos -C$\mathrm{H}$, alquenos $=\mathrm{C}-\mathrm{H}$, enlaces $-\mathrm{O}-\mathrm{H}$; dobles enlaces aromáticos $-\mathrm{C}=\mathrm{C}$-; alcoholes $-\mathrm{C}-\mathrm{OH}$ y cetonas $-\mathrm{C}=\mathrm{O}$ entre otros; de esta manera, el análisis infrarrojo permite identificar con mayor claridad los grupos funcionales presentes en el extracto.

En este sentido, el espectro infrarrojo IR - ATR, evidencia las siguientes señales importantes: una la banda ancha ubicada entre 3323 y $3294 \mathrm{~cm}^{-1}$ propia del enlace de hidrógeno asociado al oxígeno, por ejemplo grupos alcohólicos (-OH), presente en las estructuras químicas de las saponinas, taninos, triterpenos, cumarinas y algunos alcaloides.

Igualmente, se identifica una banda ubicada en $2970,38 \mathrm{~cm}^{-1}$, que puede ser evidencia de un enlace de hidrógeno asociado a carbono con enlaces $\pi(=\mathrm{C}-\mathrm{H})$; presentes en las saponinas, taninos, triterpenos, alcaloides, igualmente en los flavonoides, algunas cumarinas y las quinonas.

Por otra parte, en el espectro infrarrojo se observan bandas en 2929,87 y $2875,86 \mathrm{~cm}^{-1}$, representativas del enlace carbono hidrógeno de los alcanos $-\mathrm{C}-\mathrm{H}$, presente en todos los metabolitos identificados en el tamizaje fitoquímico.

Igualmente, se evidencia una señal a 1645,28 $\mathrm{cm}^{-1}$ característica de dobles enlaces entre el carbono y el oxígeno $-\mathrm{C}=\mathrm{O}$; bandas cercanas a $1100 \mathrm{~cm}^{-1}$ propias del enlace carbono oxígeno -C-O, especialmente del grupo funcional alcohol; finalmente, se observan señales entre 920 y 700 $\mathrm{cm}^{-1}$ las cuales son propias de las flexiones $\mathrm{y}$ estiramientos de enlaces bencílicos [30,31,32], todas estas señales ratifican las estructuras de los compuestos identificados en el tamizaje fitoquímico. De esta manera, el análisis infrarrojo permite corroborar la presencia de diversos grupos funcionales característicos de las estructuras químicas de los compuestos extraídos mediante maceración hidroalcohólica de la caléndula, corroborando el alto grado de complejidad de esta matriz.

\section{Identificación de compuestos}

El tamizaje fitoquímico del extracto hidroalcohólico de la caléndula, y la caracterización estructural demuestran las grandes posibilidades como antioxidante o posible bactericida, por lo que se justifica identificar los compuestos presentes, mediante cromatografía de gases acoplada a masas, el resultado demuestra la presencia de más de 50 compuestos diferentes. En la Tabla 3 se los compuestos identificados por espectrometría de masas y que cuentan con un potencial antimicrobiano, reafirmando la riqueza química del extracto hidroalcohólico de la caléndula, por lo tanto, su gran potencial fitoquímico como fuente de compuestos bioactivos para diversos usos. 
Tabla 3. Algunos compuestos identificados por GC-MS.

\begin{tabular}{|c|c|c|}
\hline Compuesto & Grupo funcional & Estructura química \\
\hline 5-(hidroximetil)oxolan-2-ona & $\begin{array}{l}\text { Cetona heterocíclica } \\
\text { (Lactona) }\end{array}$ & \\
\hline $\begin{array}{l}\text { 3,5-dihidroxi-6-metil-2,3-dihidro-4-ona (2,3-dihidro- } \\
\text { 3,5-dihidroxi-6-metil-4H-piran-4-ona) }\end{array}$ & Piranona & \\
\hline 2-Hidroxi-2-ciclopenten-1-ona & Cetona-alcohol & \\
\hline 4,4-dimetil-1-fenilpyrazolidin-3-ona & Cetona-Amina aromático & \\
\hline (2R)-2- Feniloxirano & Éter aromático & \\
\hline (3S)-3,5-dihidróxi-6-metil-2,3-dihidropiran-4-ona & Cetona-alcohol-eter & \\
\hline Ácido 4-hidroxi-3-metoxibenzoico (Ácido vainíllico) & Ácido-eter-alcohol & \\
\hline Alloaromadendreno & Hidrocarburo & \\
\hline
\end{tabular}

Como se observa en la Tabla 3, uno de los compuestos identificados es el 5-(hidroximetil) oxolan-2-ona, el cual se puede utilizar como un aditivo alimentario, por sus propiedades aromatizantes. Esta furanona ha sido encontrada en algunas fuentes naturales como la papaya, duraznos, piña, mango, uchuva, espárragos y té negro [33].

Igualmente, se identificó al 3,5-dihidroxi-6-metil2,3-dihidro-4-ona(2,3-dihidro-3,5-dihidroxi-6-meti$4 \mathrm{H}$-piran-4-ona), una piranona encontrada en el extracto, cuyo espectro de masas se muestra en la Figura 2. 
Esta 2,3-Dihidro-3,5-dihidroxi-6-metil-(4H)-piran4-ona ha sido encontrada como producto de procesos térmicos en gramíneas, zanahorias, cebollas, tomates y carnes, gracias a las reacciones de las aminas primarias, aminoácidos y proteínas presentes, lo cual explica el aumentando de la vida útil de estos alimentos, al someterlos a tratamientos térmicos [34, 35]. Igualmente, se le ha adjudicado características antioxidantes [36], por lo que, estos resultados obtenidos por otros investigadores, permiten suponer que el extracto hidroalcohólico de la caléndula puede ser utilizado como aditivo alimentario, con propiedades antioxidantes.

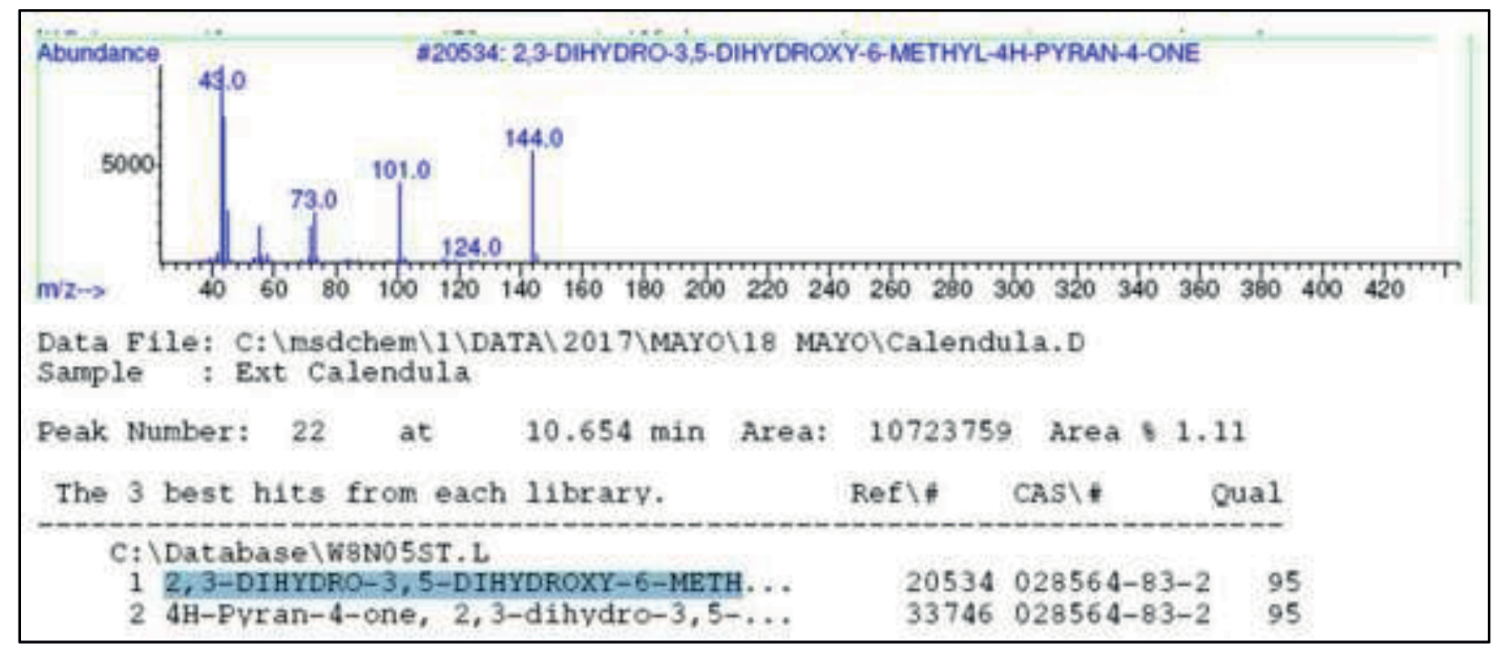

Figura 2. Espectro de masas 2,3,-Dihidro-3,5-dihidroxi-6-metil-4H-piran-4-ona.

Otro compuesto importante, presente en el extracto de caléndula, es el alloaromadendreno, una cetona cíclica que hace parte de aceites esenciales, al cual se le han identificado características antidermatofíticas y antimicrobianas, algunas investigaciones han determinado su concentración mínima inhibitoria sobre Klebsiella pneumoniae, productora de betalactamas de espectro extendido [37].

\section{Actividad antimicrobiana}

Teniendo en cuenta los resultados obtenidos en la caracterización fitoquímica, espectrometría IRATR y la identificación de compuestos mediante cromatografía de gases acoplada a espectrometría de masas, con los cuales se confirma la presencia de compuestos cetónicos, ácidos, alcoholes y éteres entre otros, es posible suponer que el extracto hidroalcohólico de caléndula cuenta con propiedades antibacterianas $[10,19]$.

De esta manera, los resultados de la prueba de efectividad antibacteriana, del extracto hidroalcohólico de caléndula, frente a los microorganismos indicadores como: Candida albicans, Pseudomonas aeruginosa $y$ Staphylococcus aureus se presentan en la Tabla 4. Los resultados obtenidos muestran una reducción del número de unidades formadoras de colonia (ufc/mL) en los tres microorganismos indicadores ensayados, con una mayor incidencia en el Staphylococcus aureus, y un menor efecto para la Candida albicans, estos resultados permiten suponer que, el extracto hidroalcohólico de caléndula se perfila como un producto natural con buenas características antibacterianas, enfocado a productos de la industria alimentaria. Cabe resaltar que, estudios anteriores reportan una buena actividad antibacterial de los extractos de caléndula aplicados en tratamientos orales $[10,12]$ por lo que, estos resultados son prometedores para diversas investigaciones.

En este sentido, el efecto del extracto sobre el Staphylococcus aureus se puede explicar teniendo en cuenta que, es una bacteria gram positiva, las cuales se caracterizan porque la pared celular no tiene una membrana externa, y se ha comprobado que los compuestos antimicrobianos $\beta$-lactámicos, como el (S)-(+)- Dihidro-5- (hidroximetil)$2(3 \mathrm{H})$ - furanona, identificado en el extracto de la caléndula, se unen a las proteínas de unión penicilina (PBP), responsables de la síntesis de la pared celular, debilitando las paredes celulares, con la consecuente lisis y muerte de la célula [38]. 
Tabla 4. Ensayo de efectividad antimicrobiana.

\begin{tabular}{|c|c|c|c|c|c|}
\hline \multirow{2}{*}{$\begin{array}{l}\text { Organismo } \\
\text { prueba }\end{array}$} & \multirow{2}{*}{$\begin{array}{l}\text { Suspensión } \\
\text { bacteriana }\end{array}$} & \multicolumn{4}{|c|}{ Recuentos viables ufc/mL } \\
\hline & & $5 \mathrm{~min}$. & $10 \mathrm{~min}$. & $15 \mathrm{~min}$. & $\begin{array}{l}\text { Desviación } \\
\text { estándar } \mathbf{s} \pm^{*}\end{array}$ \\
\hline $\begin{array}{l}\text { Pseudomonas } \\
\text { aeruginosa } \\
\text { ATCC } 15442\end{array}$ & $3,2 * 10^{8}$ & $6,0 * 10^{4}$ & $5,8 * 10^{4}$ & $6,2 * 10^{4}$ & 2 \\
\hline $\begin{array}{c}\text { Staphylococcus } \\
\text { aureus ATCC } \\
6538\end{array}$ & $4,2 * 10^{8}$ & $5,7 * 10^{4}$ & $5,5^{*} 10^{4}$ & $6,0 * 10^{4}$ & 2 \\
\hline $\begin{array}{c}\text { Candida } \\
\text { albicans ATCC } \\
14053\end{array}$ & $1,70 * 10^{7}$ & $6,1 * 10^{4}$ & $5,8 * 10^{4}$ & $6,2 * 10^{4}$ & 2 \\
\hline \multicolumn{6}{|c|}{ Reducción de la viabilidad a la concentración de la prueba } \\
\hline \multicolumn{2}{|c|}{$\begin{array}{c}\text { Pseudomonas aeruginosa ATCC } \\
15442\end{array}$} & $5,3 * 10^{2}$ & $5,5^{*} 10^{2}$ & $5,2 * 10^{2}$ & 2 \\
\hline \multicolumn{2}{|c|}{$\begin{array}{c}\text { Staphylococcus aureus ATCC } \\
6538\end{array}$} & $7,4 * 10^{2}$ & $7,6 * 10^{2}$ & $7,0 * 10^{2}$ & 2 \\
\hline \multicolumn{2}{|c|}{ Candida albicans ATCC 14053} & $2,79 * 10^{1}$ & $2,93 * 10^{1}$ & $2,74 * 10^{1}$ & \\
\hline
\end{tabular}

* Los datos son los reportados por el laboratorio que prestó los servicios de análisis

Por otro lado, el efecto sobre la Pseudomonas aeruginosa se puede explicar, por la acción de la (S)-(+)- Dihidro-5- (hidroximetil)-2(3H)- furanona, teniendo en cuenta que, las pseudomonas son bacterias gram negativas, caracterizadas por contar con una pared celular más compleja que las gram positivas, ya que cuentan con una membrana celular externa que sirve como la principal barrera de permeabilidad, la cual ayuda a retener las proteínas en el espacio perisplásmico, además, cuentan con porinas, las cuales son proteínas que forman unos canales de agua y facilitan el transporte de nutrientes dentro de la célula, para el caso de las moléculas antimicrobianas, los compuestos $\beta$-lactámicos entran por estos canales y se unen a las proteínas de unión penicilina (PBP), afectando la pared celular y la posterior lisis de la célula [38].

Por otro lado, la Candida albicans es un hongo diploide asexual (forma de levadura) y saprófito, que se encuentra ampliamente difundido en la naturaleza, se han podido aislar de alimentos, vegetales, agua, etc. así como en piel y mucosas de animales y humanos [39]. Se han encontrado diversos compuestos químicos que actúan sobre este hongo, por ejemplo las aril-fenil-cetonas, las cuales actúan sobre proteínas motoras y del citoesqueleto, específicamente sobre la actina, la miosina y la fimbrina; los hidrocarburos terpénicos, alcoholes terpénicos y fenoles terpénicos, los cuales actúan sobre el transporte o síntesis de lípidos que afectan la función e integridad de la membrana celular [40].

De esta manera, se presume que los triterpenos identificados en el extracto hidroalcohólico de la caléndula, son compuestos químicos que actúan sobre la Candida albicans, generando una disrupción celular, por otra parte, las quinonas, igualmente presentes en el extracto, pueden actuar como fungicida, debido a su actividad de contacto multisitio, o los hidrocarburos aromáticos, como el caso de la (2R)-2- Feniloxiran, los cuales pueden actuar en la peroxidación de la célula, igualmente, la presencia de 4,4-dimetil-1-fenil3-pyrazolidinona, cuya estructura similar a las amino pirazolinona, puede actuar sobre la síntesis de esterol en la membrana como un inhibidor de reductasa [40].

\section{Ensayo de aplicación sobre fresa}

Los resultados obtenidos en los análisis fisicoquímicos y microbiológicos muestran que el extracto hidroalcohólico de la caléndula tiene un buen potencial como agente antimicrobiano, razón por la cual, se justifica la realización de pruebas invivo, aplicado en aspersión sobre fresas, en la Figura 3 se muestra el seguimiento fotográfico del ensayo sobre la vida útil de la fresa.

El ensayo sensorial cualitativo exploratorio se realizó por triplicado en condiciones ambientales, 
temperatura de $20^{\circ} \mathrm{C}$ y humedad relativa de $70 \%$, lo cual permitió observar el efecto en la vida útil de la fresa (Fragaria chiloensis L.), a unas condiciones favorables para el crecimiento de hongos.
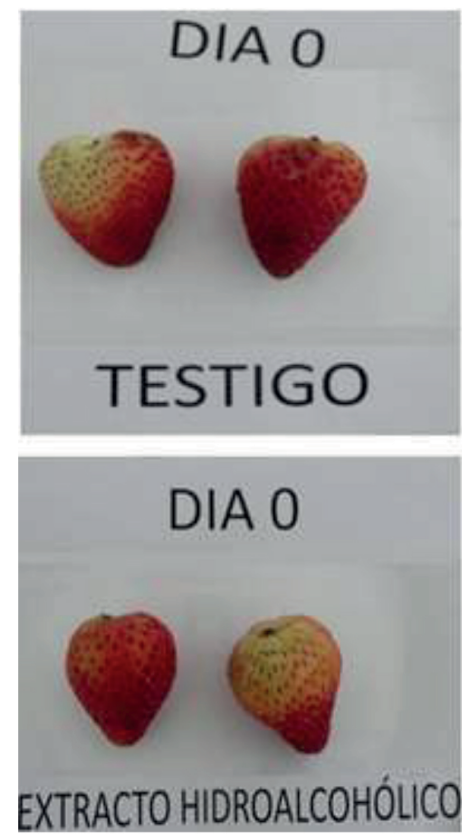
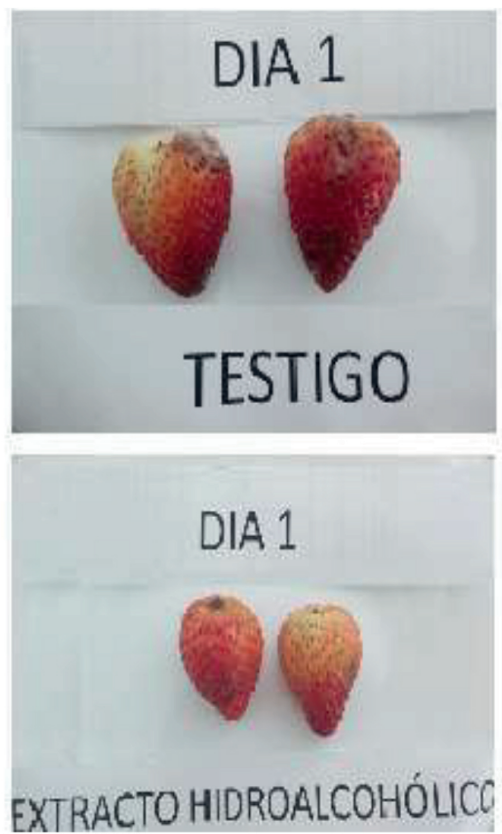
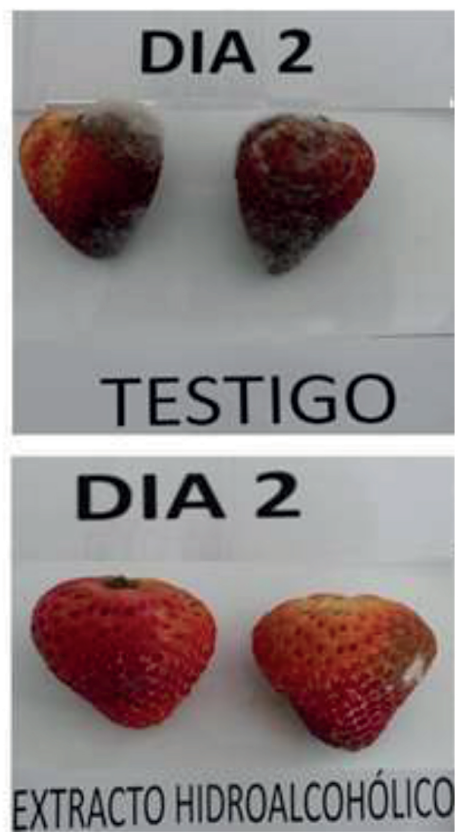

Figura 3. Efecto sobre la vida útil de la fresa, al aplicar el extracto de la caléndula.

Como se puede observar en la figura anterior, las fresas testigo, aquellas a las cuales no se les aplicó el extracto, evidencian la aparición de las primeras colonias de hongos un día después de iniciar el ensayo, y un alto crecimiento de hongos después a los dos días del inicio de la prueba.

Por otra parte, en el caso de las fresas tratadas con el extracto de caléndula, las primeras colonias visibles, a las mismas condiciones ambientales que las fresas testigo, aparecen al segundo día después de iniciar la prueba, por lo que, se presume que el extracto permite aumentar en un día la vida útil del producto.

\section{Conclusiones}

La extracción de compuestos químicos presentes en la flor de la caléndula, mediante el método de percolación con una solución hidroalcohólica, permitió obtener una mezcla química compleja, en la cual, mediante un análisis cualitativo fitoquímico, fue posible identificar la presencia de metabolitos como flavonoides, saponinas, taninos, triterpenos, alcaloides, carotenoides, cumarinas y quinonas, algunos de ellos se les ha reconocido por sus propiedades antioxidantes, antibacterianas $y$ antifúngicas.
La caracterización estructural, realizada mediante análisis infrarrojo, permitió identificar bandas características de alquenos, alcanos, compuestos bencílicos, así como bandas propias de grupos funcionales como: alcoholes y carbonilos, propios de los metabolitos identificados en el análisis fitoquímico.

El uso de cromatografía de gases acoplada a masas, permitió separar e identificar más de 50 compuestos químicos presentes en el extracto de la caléndula, entre los que se pueden encontrar: furanonas, cetonas, ácidos, ésteres e inclusive feromonas, entre muchos otros componentes, sin embargo, en la revisión bibliográfica, solamente los ocho compuestos presentados en este artículo, se les ha encontrado actividad antimicrobiana.

La actividad antimicrobiana, determinada mediante el ensayo de efectividad antimicrobiana en colonias de microorganismos indicadores, evidenció que el extracto hidroalcohólico reduce la presencia de colonias, especialmente para el Staphylococcus aureus.

El ensayo de aplicación directa por aspersión sobre fresas, mostró el posible potencial del extracto hidroalcohólico de la caléndula, como aditivo para prolongar su vida útil, teniendo en cuenta que la aparición de las primeras colonias de hongos se 
observaron después de dos días de iniciado el ensayo, a condiciones ambientales.

Los resultados de la presente investigación sugieren que el extracto hidroalcohólico de la caléndula, tiene un gran potencial como sustancia natural antibacterial, la cual puede ser utilizada en la industria alimentaria, con el fin de prolongar la vida útil de alimentos perecederos, por lo que se recomienda ampliar la investigación, variando la forma de aplicación del extracto en los alimentos, así como, ensayando otros procesos de extracción.

\section{Agradecimientos}

Los autores expresan sus agradecimientos a la Universidad Pedagógica Nacional, Facultad de Ciencia y Tecnología, Departamento de Química, así como a Kolbe Internacional SAS., por facilitar sus laboratorios, materiales, reactivos y equipos, sin los cuales no hubiese sido posible la realización de la presente investigación.

\section{Referencias bibliográficas}

[1] Vladislav O. Antioxidante and antimicrobial propierties of oregano extract. Foods and Raw Material. 2020 8(1):84-90.

[2] Dominguez E. Efecto de la aplicación del extracto hidroalcohólico de flores de caléndula (Calendula officinalis) en la estabilización del color y vida útil en pulpa de frutas(Tesis Maestría) . Bogotá, Colombia, Universidad Nacional de Colombia. Bogotá: Universidad Nacional de Colombia; 2012.

[3] Alenisan M, Alqattan $H$, Tolbah L, Shori A. Antioxidante properties of dairy products fortified with natural additives. Journal of the Association of Arab Universities for Basic and Applied Sciences. 2017: 101-106.

[4] Barreira J, Ferreira I, Oliveira B, Pereira J. Antioxidant activities of the extracts from chestnut flower leaf, skins and fruit. Food chemistry. 2008; 107(3): 1106-1113.

[5] Valdés $A$, Ramos $M$, Beltrán $A$, Jiménez $A$, Garriós M. State of the art of antimicrobial edible coatings for food pakaging applications. Coating. 2017; 7(56): 2-23.

[6] Organización de las Naciones Unidas para la Alimentación y la Agricultura (FAO). CODEX ALIMENTARIUS. Washington, USA; 1995

[7] Castro H, Parada F. Evaluación del efecto protector contra la oxidación lipídica de fracciones obtenidas a partir del pericarpio de tomáte de arbol (Solanum betaceum sendtn). Revista Colombiana de Químic. 2017, 46(2): 17-23.

[8] Paim M, Maciel $M$, Weschenfelder $S$, Bergmann G, Avancini C. Anti-Echerichia coli effect of Hibiscus Sabdariffa L. in a meat model. Food Dcience and Technology. 2017; 37(4): 647-650.

[9] Águila B, Menéndez R, González C, Fernández D. Extracto acuoso de Calendula officinalis, estudio preliminar de sus propiedades. Revista Cubana de Plantas Medicinale. 2000; 5(1): 30 31.

[10] Shirinidhi S, Soumya B, Rukmini J, Chethana Kala B, Shiv K. Efficacy of calendula officinalis extract (Marigold flower) as antimicrobial agent against oral microbes: An invitro study in comparision with chlorexidine digloconate. Journal of Clinical and Diagnostic Researc. 2017; 11(10): 5-10.

[11] Meyer G, Sarmiento O, Ramirez R, Guevara O. Estimación del contenido de fenoles totales en aceite esencial de caléndula (Calendula officinalis L.) Obtenido mediante OAHD. Revista ION. 2018; 31(1): 7-12.

[12] Amira E, Ghada S, Abeer A, Heba E. Prevention of bacterial biofilm formation on soft contact lenses using natural compounds. Journal of Ophthalmic Inflamation and Infection. 2017; 7(11): 1-7.

[13] Cámara de Commercio de Bogotá. Manual de fresa. Bogotá, Colombia; 2015.

[14] Valgas C, Simone M, Elza F, Smänia J. Screening methods to determine antibacterial activity of natural products. Brazilian Journal of Microbiology. 2007; 38(2): 369-380.

[15] Varela M, Maekawa L, Oliveira L, Cardoso A, Shygei E, Carvalho C. In vitro antimicrobial activity of auxiliary chemical substances and natural extracts on Candida albicans and Enterococcus fecalis in root canals. Journal of Applied Oral Science. 2013; 21(2): 118-123.

[16] Acosta L, Rodríguez C, Sánchez E. Instructivo técnico de Calendula officinalis L. Revista Cubana de Plantas Medicinales. 2001; 1: 2327.

[17] Sánchez G, Castro C, Álvarez G, Flores J, Barriga M. Compuestos fenólicos y actividad antioxidante de los extractos de la hoja de chirimoya (Annona cherimola Mill). Revista Colombiana de Química. 2019; 48(2): 21-26.

[18] Ortiz L, Chaves G. Composición fitoquímica del extracto de raíz de Ichthyothere terminalis 
de dos regiones geográficas de Colombia. Revista Colombiana de Química. 2017; 46(3): 11-16.

[19] Lima B, Oliveira R, Santos E, Bittencourt M, Santos O. Phytochemical characterization and bioactivity of ethanolic extracts on eggs of citrus blackfly. Ciência Rural. 2017; 47(11): 1-6.

[20]Aparicio R, Velasco J, Paredes R, Rojas L. Caracterización química y actividad antibacteriana del aceite esencial de Manguifera indica L. de tres regiones de Venezuela. Revista Colombiana de Química. 2019; 48(3): 13-18.

[21] Instituto Colombiano de Normas Técnicas. Antisépticos y desinfectantes químicos actividad bactericida básica. Método de prueba y requisitos (fase 1). Bogotá, Colombia: ICONTEC; 2003.

[22] Casierra F, Peña J, Vargas A. Propiedades fisicoquímicasde fresa (Fragaria $\mathrm{sp}$ ) cultivadas bajo filtros fotoselectivos. Revista Facultad de Agronomía Medellín. 2011; 64(2): 6221-6228.

[23] Vicente O, Bascaiu M. Flavonoids antioxidand compounds for plant defence and for a healthy human diet. Notulae Botanicae Horti Agrobotanici Cluj-Napoca. 2018; 46(1): 14-21.

[24] Han L, Yao S, Cao S, Mo G, Li J, Cao Y, et. al. Triterpenoid saponins from Anemone faccida suppress tumor cell proliferation by regulating MAPK, PDI/PDLI, and STAT3 signaling pathways and altering cancer metabolism. Onco Targets and Therapy. 2019; 12: 1091710930.

[25] Hussain G, Huang J, Rasul A, Anwar H, Imran A, Maqbool J, et.al. Putative roles of plantderived tannins in neurodegenerative and neuropsychiatry disorders and update review. Molecules. 2019; 24(12): 2-16.

[26]Kochan E, Szymanska G, Grzegorczy I, Szymczyk P, Sienkiewicz M. Ginsenoside and phenolic compounds in hydromethanolic extracts of American ginseng cell cultures and their antioxidant properties. Acta Societatis Botanicorum Poloniae. 2019; 88(4): 1-11.

[27] Payili N, Yennam S, Rekula S, Naidu C, Bodde $\mathrm{Y}$, Ghosh B. Design synthesis and evaluation of anticancer properties of novel quinone bearing Charbamyl $\beta$-Lactam hybrids. Journal of Heterocyclic Chemistry. 2018; 55(6): 13581365.

[28] Espada L, Ferrer A, Batista Y, Bandera A, Almeida Y. Separación y clasificación de los alcaloides presentes en las hojas y flores de la especie Sipilantes urens Jacq. Revista Cubana de Química. 2016; 28(1): 409-430.

[29] Heredia L. Programa guia de actividades para la enseñanza de conceptos asociados a la inhibición enzimática a través del docking molecular en ambientes de aprendizaje blenden learning (Tesis). Bogotá, Colombia: Universidad Pedagógica Nacional; 2019.

[30]Calderón G. Manual para la interpretación de espectros infrarrojos. Bogotá: Universidad Nacional de Colombia; 1985.

[31] Pretsch E, Clerc T, Seibl J, Simon W, Castells J, Camps F. Tablas para la elucidación estructural de compuestos orgánicos por métodos espectroscópicos. 2 ed. Madrid: Editorial Alhambra; 1985.

[32] Silverstein R, Webster F, Kiemle D, \& Bryce D. Spectrometric identification of organic compounds. 8 ed. New Jersey: Wiley; 2014.

[33] National center for Biotechnology Information. PubChem (sitio en internet). NCBI. Disponible en: https://pubchem.ncbi.nlm.nih.gov/ . Acceso el 29 de Septiembre 2019.

[34]Mozafari A, Vafaee $Y$, Shahyad M. Phytochemical composition and invitro antioxidant potential of Cynodon dactylon leaf and rhizome extracts as affected by dryiong methods and temperatures. Journal of Food Science and Technology. 2018; 55(6): 20202029.

[35] Ledl F, Schnell W, Severin T. Nachweis von 2,3-Dhydro-3,5-dihydroxy-6-methyl-4Hpyran-4-on in lebensmitteln. Zeitschrift für Lebensmittel-Untersuchung und Forschung. 1976; 160(4): 367-370.

[36] Čechovská L, Cejpek K, Konečný M. On the role of 2,3-dihydro-3,5-dihydroxy-6-methyl(4H)-pyran-4-ona in antioxidant capacity of purenes. Europan Food Research and Technology. 2011; 233(3): 367-376.

[37] Morales M, Domínguez M, González G, Bello $\mathrm{H}$. Actividad antimicrobiana de aceite esencial de eucalyptus globulus, proveniente de la región del Blo-Bio Chile, sobre Klebsiella pneumonieae productoras de BLEE. XIII Congreso Argentino de Microbiología. 2013 sep 23-26; Buenos Aires, Argentina

[38] Stephen J, Harbeck R, McCarter Y, Ortez J, Rankin I, Sautter R, et.al. Manual de pruebas de susceptibilidad antimicrobiana. Seattle: University of Washington; 2005.

[39]Bazán E, Sánchez E, Córdoba E, Hernández 
F, Manzano P, López R. Hallazgo de Candida albicans en manos de manejadoras de alimentos. Revista Mexicana de Patología Clínica. 2001; 48(1): 37-41.
[40]Fungicide Resistance Action Committee. Clasificación de fungicidas y bactericidas según el modo de acción. Basilea, Suiza: FRAC; 2019. 